\title{
BONE DISEASE IN PATIENTS WITH PRIMARY SCLEROSING CHOLANGITIS
}

\author{
Paul Angulo, MD1 ${ }^{1}$, Garfield A. Grandison, MBBS ${ }^{1}$, Derek G. Fong, MD ${ }^{2,3}$, Jill C. Keach², \\ Keith D. Lindor, MD ${ }^{2}$, Einar Bjornsson, MD $^{2,4}$, and Alvaro Koch, MD $^{1}$ \\ ${ }^{1}$ Division of Digestive Diseases and Nutrition, University of Kentucky Medical Center ${ }^{2}$ Division of \\ Gastroenterology and Hepatology, Mayo Clinic Rochester, $\mathrm{MN}^{3}$ Kaiser Permanente South Bay \\ Med Center, Harbor City, CA ${ }^{4}$ Landspitali University Hospital, Reykjavik Iceland
}

\begin{abstract}
BACKGROUND \& AIMS-Osteopenic bone disease occurs frequently among patients with chronic liver disease but has not been well studied in those with primary sclerosing cholangitis (PSC). We investigated the prevalence, rate of progression, and independent predictors of bone disease in a large number of patients with all stages of PSC.
\end{abstract}

METHODS—Bone mineral density of the lumbar spine, hip, and total body was measured yearly for 10 years in 237 patients with PSC.

RESULTS-Osteoporosis (t-score below -2.5) was found in 15\% of patients and occurred 23.8fold (95\% confidence interval [CI]: 4.6-122.8) more frequently in those with PSC than expected from a matched population. By multivariate analysis, age $\geq 54$ years (odds ratio $[\mathrm{OR}]=7.8,95 \%$ CI: 3.3-18.3], body mass index $\leq 24$ ( $\mathrm{OR}=4.9,95 \%$ CI: $1.9-12.6)$, and inflammatory bowel disease for $\geq 19$ years (OR=3.6, 95\% CI:1.5-8.4) correlated with the presence of osteoporosis. Osteoporosis was present in $75 \%$ of patients with all 3 risk factors, but in only $3.1 \%$ of those without all of them. Patients with PSC lost $1 \%$ of bone mass per year; this rate of bone loss was significantly associated with duration of inflammatory bowel disease.

CONCLUSIONS-Osteoporosis occurs frequently among patients with PSC. Old age, low body mass index, and long duration of inflammatory bowel disease can be used to identify patients with PSC who might derive the most benefit from measurements of bone density and treatments for bone diseases.

\section{Keywords}

Cholestatic liver disease; autoimmune liver disease; Osteoporosis; inflammatory bowel disease

Correspondence: Paul Angulo, MD, Division of Digestive Diseases and Nutrition, University of Kentucky Medical Center, 800 Rose Street, Rm MN649, Lexington, KY 40536, (859) 323-3555, (859) 257-8860 fax, paul.angulo@uky.edu.

Disclosures: Nothing to disclose

Contribution to the manuscript

Conception and design of the study: P. Angulo

Generation, collection, assembly, analysis and/or interpretation of data: P. Angulo; G. Grandison; D. Fong; J. Keach; E. Bjornsson. Drafting or revision of the manuscript: P. Angulo; G. Grandison; D. Fong; J. Keach; E. Bjornsson; K. Lindor; A. Koch Approval of the final version of the manuscript: P. Angulo; G. Grandison; D. Fong; J. Keach; and E. Bjornsson; K. Lindor; A. Koch Publisher's Disclaimer: This is a PDF file of an unedited manuscript that has been accepted for publication. As a service to our customers we are providing this early version of the manuscript. The manuscript will undergo copyediting, typesetting, and review of the resulting proof before it is published in its final citable form. Please note that during the production process errors may be discovered which could affect the content, and all legal disclaimers that apply to the journal pertain. 
Metabolic bone disease with its predisposition to fractures and bone pain occurs commonly in patients with chronic liver disease of different causes. 1 Bone disease has been studied extensively in patients with chronic cholestatic liver disease such as primary biliary cirrhosis (PBC).2 Primary sclerosing cholangitis (PSC) is another chronic cholestatic liver disease that, unlike PBC, occurs at a younger age and affects mainly males. 3 Most patients with PSC also suffer from inflammatory bowel disease (IBD) which itself, particularly Crohn's disease significantly increases the risk of developing osteoporosis.4,5 This risk is even greater in patients with IBD treated with corticosteroids.6 Bone disease in patients with PSC, however, has remained largely unstudied.

In an early report, 15 out of 30 patients with PSC undergoing evaluation for liver transplantation were found to have severe bone disease, but bone mass was normal in 18 patients studied with recently diagnosed PSC. ${ }^{7}$ Two more recent reports that included highly selected patients with PSC found osteoporosis in $9 \%$ of patients at the time of enrollment in a randomized trial of ursodeoxycholic ${ }^{8}$ and in a third of patients with end-stage PSC evaluated for liver transplantation. ${ }^{9}$ In the early post-transplant period both the rate of bone loss and the proportion of patients experiencing fractures increased substantially with low bone mass in the pre-transplant period being one of the strongest predictors of posttransplant fractures. ${ }^{10}$

Hence, larger studies with unselected patient populations are necessary to determine the real prevalence of bone disease in patients with PSC, and to characterize those patients with more severe bone disease. Furthermore, the identification of patients with bone disease preliver transplantation would help in selecting those patients with PSC more apt to derive the most benefit from enrollment in clinical trials and treatment of their bone disease. In addition, a large study with long-term follow-up may potentially serve as the basis for future guidelines regarding the frequency of bone density testing in patients with PSC. To deal with these issues we conducted this longitudinal, cohort study designed 1) to determine the prevalence and rate of progression of bone disease in a large number of patients with all stages of PSC, and 2) to identify predictors of bone disease and progression.

\section{MATERIAL AND METHODS}

\section{Patient Population}

The bone mineral density (BMD) of the lumbar spine at L2-L4 was determined in 237 patients with cholangiography- and liver biopsy-confirmed PSC. These patients were seen at the Mayo Clinic in Rochester, MN for the first time from 1/1/1991 to 12/31/1998. The date of 1/1/91 was chosen because it was the date when the current normal values of bone density measurement were established at the Mayo Clinic. Measurement of BMD was done at the time of the first visit and, in most patients, repeated at annual intervals thereafter. BMD of left femoral neck and total body was also measured in 48 and 60 patients, respectively.

The diagnosis of PSC was established based upon the following criteria: 1) chronic cholestatic liver disease of at least 6 months' duration with serum alkaline phosphatase activity at least 1.5 times the upper limit of normal; 2) liver biopsy within 3 months consistent with the diagnosis of PSC; 3) endoscopic and/or percutaneous cholangiography showing the typical changes of PSC; and 4) appropriate exclusion of other liver diseases.

All patients had a complete medical history and physical examination performed at the time of the first bone density measurement and at regular intervals thereafter. Information about fractures was obtained both clinically and with radiographs taken at the lumbar and thoracic spines and at the site of any bone pain. Body mass index (BMI) was calculated using the 
formula: $\mathrm{BMI}=$ weight in kilograms/(height in meters)2. Hematological and biochemical parameters including hemoglobin, platelet count, prothrombin time, serum alkaline phosphatase activity, and serum levels of total bilirubin, aspartate aminotransferase (AST), albumin, calcium, phosphorus, creatinine, and vitamin D (total, 25-hydoxy, and 1,25 dihydroxy) were determined at the time of the first bone density measurement. Calcium levels were corrected for albumin levels: $0.8 \mathrm{mg} / \mathrm{dL}$ of calcium were added for every $1.0 \mathrm{~g} /$ $\mathrm{dL}$ that serum albumin fell below $4.0 \mathrm{~g} / \mathrm{dL}$. Esophagogastroduodenoscopy and ultrasonography of the abdomen were also performed. The Mayo risk score was calculated using the revised PSC score:11 Risk $=0.03 *$ age (years) $+0.54 * \log ^{\mathrm{e}}$ (total bilirubin $[\mathrm{mg} /$ $\mathrm{dL}])-0.84 *$ albumin $(\mathrm{g} / \mathrm{dL})+0.54 * \log ^{\mathrm{e}}($ AST $[\mathrm{IU} / \mathrm{L}])+1.24 *$ variceal bleeding $($ yes $=1$; no $=0$ ).

Colonoscopy and/or flexible sigmoidoscopy to assess for IBD were performed in all cases and the diagnosis of IBD was confirmed histologically. The type of IBD was recorded and cumulative lifetime prednisone dose until the date of the first bone density measurement was calculated. Disease activity at the time of bone density measurement was determined using the Truelove and Witts index for ulcerative colitis ${ }^{12}$ (index 6-19 means disease in remission; index> 19 means active disease) and the Crohn's disease activity index for Crohn's disease (index $>150$ means active disease). ${ }^{13}$ Data on surgical resection of the colon and the length of the resected small bowel were recorded in each patient. Duration of IBD was determined as the time elapsed since the first clinical, endoscopic or histologic evidence leading to the diagnosis of IBD until the time of the first bone density measurement. Duration of PSC was determined as the time elapsed since the first clinical, biochemical or cholangiographic findings leading to the diagnosis of PSC until the time of the first bone density measurement.

A detailed review of drug history and drugs taken during follow-up were recorded. During follow-up, budesonide was given to eight (3.4\%) patients for treatment of $\mathrm{PSC}^{14}$ while biphosphonates were given to $3(1.3 \%)$ patients for treatment of bone disease. However, the bone density measurements performed during and after treatment with these medications were not included in any part of this analysis.

\section{Bone Density Measurement}

Bone mineral density (BMD) was measured by dual energy $\mathrm{x}$-ray absorptiometry using Hologic machines (measurements reproducibility of $2.2 \%$ ). Bone mass was expressed in absolute values $\left(\mathrm{g} / \mathrm{cm}^{2}\right)$, t-score (number of standard deviations compared with a young [30year old] adult sex-matched reference population), and z-score (number of standard deviations compared with an age-and sex-matched reference population). As defined by the World Health Organization, a t-score between -1 and -2.5 would indicate osteopenia, while a t-score below -2.5 would indicate osteoporosis. ${ }^{15}, 16 \mathrm{~A}$ z-score below -2 would indicate a value in the lowest $2.5^{\text {th }}$ percentile of the reference range, a value associated with a considerably larger increase in the risk of fracture. ${ }^{17}$

\section{Matched Control Population}

Bone density measurements were compared with the expected normal bone density adjusted for age, sex, and ethnic group using standard values for normal United States population provided by the Hologic reference database (Hologic, Inc; Waltham, MA) ${ }^{18,} 19$ This database contains nearly 1000 lumbar spine and over 1400 hip measurements performed in healthy adult volunteers of either sex.18,19 


\section{Statistical Analysis}

Comparison of clinical, biochemical and histologic variables was done across t-scores and zscores. Results are expressed as mean \pm SEM (range) and number (proportion) of patients with a condition. The risk of having bone disease ( $\mathrm{t}$-score below -2.5 ; $\mathrm{z}$-score below -2 ) was calculated using the corresponding probability of the standard normal density, that is, $\mathrm{P}(\mathrm{t}<-2.5)$ or $\mathrm{P}(\mathrm{z}<-2)$. Univariate analysis was performed using standard parametric (if normally distributed) or non-parametric (if non-normally distributed) statistics for comparison of continuous variables. The Chi-squared or Fisher's Exact test, where appropriate, was used for comparison of frequency data. The independent effect of significant variables $(\mathrm{p}<0.05)$ on the severity of bone disease was assessed using multivariate logistic regression analysis with both the backward and forward stepwise selection procedures. Receiver operating characteristics curve (ROC) were created to identify the best cut-off value of independent predictors to distinguish between patients with and without bone disease. Multivariate linear regression analysis was used to identify independent predictors of the rate of progression of bone disease. The study was approved by the Mayo Institutional Review Board and all patients had given informed consent for participation in medical research.

\section{RESULTS}

Table 1 summarizes the demographic, clinical and biochemical data as well as the histologic stage of disease of the patient population. The 237 patients, including $100(42 \%)$ female and 137 (58\%) male had a mean age of $45.5 \pm 0.8$ years (range 15-73). One-hundred seventyfive (74\%) patients had IBD including 142 (60\%) patients with ulcerative colitis, 32 (13.4\%) with Crohn's disease, and one (0.6\%) patient with unclassified IBD. The bowel disease was in remission in all patients at the time of the first bone density measurement. About a half of patients had earlier (stage 1-2) disease, and 52 (22\%) patients had cirrhotic-stage PSC. Deficiency of vitamin D and calcium was found in $54(23 \%)$ and $9(4 \%)$ patients respectively and corrected by oral supplementation. Prior fractures occurred in 14 (5.9\%) patients and, in all of them, the t-score was in the range of osteopenia or osteoporosis.

At baseline, the bone density of the lumbar spine correlated strongly with the bone density of the hip $(\mathrm{r}=0.65, \mathrm{P}<0.001)$ and with that of the total body $(\mathrm{r}=0.78, \mathrm{P}<0.001)$. The baseline bone density of the lumbar spine was significantly lower in PSC than expected in a matched population of the same age and sex $\left(0.97 \pm 0.01 \mathrm{vs} .1 .05 \pm 0.004 \mathrm{~g} / \mathrm{cm}^{2}\right.$ respectively, $\mathrm{p}<0.001)$ as was the t-score $(-1.19 \pm 0.09$ vs. $-0.43 \pm 0.03$ respectively, $\mathrm{p}<$ $0.001)$. There was no a significant difference between patient with and without IBD in terms of baseline bone density $(\mathrm{p}=0.8)$, t-score $(\mathrm{p}=0.6)$, and $\mathrm{z}$-score $(\mathrm{p}=0.3)$.

\section{Prevalence of bone disease}

A t-score below -2.5 (osteoporosis) of either the lumbar spine or femoral neck was found in $35(15 \%)$ patients; this prevalence of osteoporosis was 23.8 times [95\% confidence intervals (CI) 4.6-122.8] higher in patients with PSC than expected in a young adult sex-matched population. Ninety-seven (41\%) patients had a t-score between -1 and -2.5 (osteopenia), and 105 patients (44\%) had a t-score above -1 (normal bone mass). Severe osteoporosis (zscore below -2) of either the lumbar spine or femoral neck was found in $33(14 \%)$ patients; this prevalence of severe osteoporosis was 6.1 times (95\% CI 2.5-14.9) higher in patients with PSC than expected in an age- and sex-matched population.

\section{Predictors of t-score}

Table 1 summarizes the univariate analysis of the association of osteoporosis and potential predictors. By multivariate analysis only age, BMI and duration of IBD were significantly 
associated with the presence of osteoporosis. Based on ROC curves the best threshold for age was 54 years, for BMI was 24, and for duration of IBD was 19 years. Table 2 summarizes the odds ratio and $95 \% \mathrm{CI}$ for the three factors independently associated with osteoporosis. When age was excluded from the model, the menopausal status gained statistical significance [odds ratio (OR) 4.04, 95\% CI 1.44-11.34, P = 0.008]. Seventy-five percent of patients 54 years or older who had a BMI equal to or less than 24 as well as 19 years or more of IBD duration had osteoporosis. However, only $3.1 \%$ of patients younger than 54 years, with a BMI greater than 24 and less than 19 years of IBD duration had osteoporosis (Figure 1). The presence of only one, or two of these three risk factors predicted osteoporosis in $12.5 \%$ to $47 \%$ of our cohort.

\section{Predictors of z-score}

Table 1 summarizes the results of the univariate analysis of the association of severe osteoporosis and potential predictors. By mutivariate analysis only BMI and duration of IBD correlated significantly with the presence of severe osteoporosis (Table 3).

\section{Rate of Progression of bone disease}

One-hundred and eight (46\%) patients were eligible for this part of the study. These patients had a total of 568 bone densities of the lumbar spine, 88 of the femoral neck, and 126 of the total body measured during a mean follow-up of $4.0 \pm 0.2$ years (range 1-10 years). The remaining 129 patients had only the baseline bone density measurement. During follow-up, the bone density of the lumbar spine correlated significantly with the bone density of the hip in 88 paired measurements and with that of the total body in 126 paired measurements (Figures 2A and 2B).

Patients with PSC lost bone mass at an average rate of $-0.010 \pm 0.002 \mathrm{~g} / \mathrm{cm}^{2} /$ year (range -0.10 to $0.04 \mathrm{~g} / \mathrm{cm}^{2} / \mathrm{year}$ ), that is, an average of $1 \%$ bone mass per year (Figure 3 ). This rate of bone loss was similar in the three bone sites evaluated $(p=0.7)$. This rate of bone loss in PSC was significantly higher than the rate of bone loss expected in a matched population of the same age and sex $\left(-0.010 \pm 0.002\right.$ vs. $-0.004 \pm 0.000 \mathrm{~g} / \mathrm{cm}^{2} /$ year, respectively, $\mathrm{p}=$ $0.03)$. The rate of bone loss in PSC was not significantly affected by vitamin $D$ and/or calcium supplementation ( $p=0.5)$, use of corticosteroids $(p=0.2)$ or hormone replacement therapy $(\mathrm{p}=0.4)$. By univariate analysis duration of IBD, alkaline phosphatase, AST, triglycerides and Mayo risk score were significantly associated with a greater rate of bone loss. By multivarate analysis, however, the only variable that correlated significantly with the rate of loss of bone mass was a longer duration of IBD $(p=0.02)$ even when hormone replacement therapy, use of corticosteroids and vitamin $\mathrm{D}$ and calcium supplementation were included in the model.

\section{DISCUSSION}

In this large series, we found that $15 \%$ of patients with PSC had established osteoporosis (t score $<-2.5)$ at the time of their first visit to our institution with a 23.8 -fold increased risk of osteoporosis. Fourteen percent had severe osteoporosis (z score $<-2.0)$ at the time of first visit with a 6.1 -fold increased risk of severe osteoporosis. Using routinely determined clinical, biochemical and histological variables we have shown that factors that independently predict the presence of osteoporosis are advanced age, lower BMI and longer duration of IBD. Patients with PSC lost bone mass at a rate of $1 \%$ per year and this rate of bone loss over time seemed to be greatly influenced by the duration of IBD.

Our prior report on bone disease in PSC included only 81 patients, seven of whom had osteoporosis, followed-up for a short period of time. ${ }^{8}$ As such, we were not able to perform 
robust multivariate analyses looking at predictors of bone disease and rate of bone loss in PSC. In addition, since those patients were selected from participants enrolled in a clinical trial of ursodeoxycholic acid, we were not able to determine a realistic estimate of the prevalence and severity of bone disease in PSC. ${ }^{8}$ Hence, all conclusions in that study were drawn on the basis of simple univariate comparison between patients with and without severe bone disease. The current study is powered and designed to allow us to draw more meaningful and reliable conclusions regarding bone disease in PSC.

Advanced age and lower BMI are well-known risk factors for the development of osteoporosis in an otherwise healthy population as is menopausal status. ${ }^{20}$ Our findings of the correlation between advanced age and lower BMI with osteoporosis in patients with PSC may be related to similar pathophysiologic mechanisms which occur in the general population. Osteoporosis is one of the few conditions in which excess body weight is protective $^{21,22}$ Intriguing are recent results suggesting that the polypeptide hormone leptin may modulate bone mass indirectly by a central effect. ${ }^{23}$ This finding may provide a molecular explanation for the relationship between body weight and osteoporosis.

In our study, although menopausal status was not initially significant by multivariate analysis, this variable was significant as an independent predictor of osteoporosis when age was excluded from the model which indicates that the effect of menopausal status as an independent predictor of osteoporosis is captured by age when both variables compete in the model.

PSC is often referred as a disease affecting predominantly men; however, $42 \%$ of our cohort were women which simply reflects the changes in the demographics of this disease over the last four decades. For instance, the proportion of women with PSC seen in our institution was only $30 \%$ in the 1970 ' $\mathrm{s}^{24}$ as compared with a proportion greater than $40 \%$ in the last two decades (unpublished data). Interestingly, neither the severity of bone disease nor the rate of boss loss was significantly affected by gender in our cohort.

The 15\% prevalence of osteoporosis found in our patients with PSC is lower than the prevalence of $20 \%$ to $37 \%$ reported in patients with PBC..$^{25-28}$ In patients with PBC, older age, lower BMI, menopausal status, and more advanced liver disease were independently correlated with presence of osteoporosis. ${ }^{25,28}$ The exact pathogenic mechanism for osteopenic bone disease in chronic cholestatic liver disease remains unknown, but cholestasis on its own may contribute significantly to bone loss in patients with PSC and PBC. Bilirubin has been shown to inhibit osteoblast function in vitro ${ }^{29}$ and this osteoblast dysfunction may play a role in the low bone formation rate seen in cholestatic patients. Higher serum bilirubin and other surrogate markers of disease severity such as albumin, Mayo risk score and histologic stage of disease were significantly associated with the presence of osteoporosis by univariate analysis in our patients with PSC. However, unlike PBC the severity of liver disease as determined by these factors seemed to be of less importance when these variables competed with age, BMI, duration of IBD and menopausal status in a multivariate model. Intriguing are recent data demonstrating similar bone histomorphometric changes in PSC and PBC suggesting that both decreased bone formation and increased resorption contribute to bone disease in female patients with PSC or PBC, whereas decreased bone formation rather than increased resorption may be an important pathogenic mechanism in male patients with these conditions. ${ }^{30}$ These histomorphometric studies were done in patients with advanced PSC and PBC undergoing liver transplantation, 30 and thus further studies are necessary to determine whether similar histomorphometric changes occur in patients with earlier stages of liver disease. 
Treatment with corticosteroids is a well-known risk factor for development of osteoporosis in patients with IBD.4, 6 The use of corticosteroids in IBD is usually determined by the severity of bowel symptoms. Hence, an intriguing observation in our study is the lack of correlation between lifetime cumulative dose or use of corticosteroids and severity of bone disease. This may be explained by the fact that IBD associated with PSC generally follows a more quiescent course than in patients without PSC and hence, the lifetime dose of corticosteroids for the treatment of IBD in PSC may be lower than in patients without underlying PSC. ${ }^{31}$ In fact, only $25 \%$ of our patients required corticosteroid therapy at some point (20\% for the treatment of IBD); this proportion is much lower than that reported in patients with IBD without PSC $^{31-} 33$ Hence, although corticosteroids are not recommended for the treatment of PSC, the use of corticosteroids for the treatment of IBD seems to be necessary in only a small proportion of patients with PSC, and this may explain the lack of significant detrimental effects of corticosteroids on bone mass in our cohort of patients with PSC. The longer duration of IBD which correlated significantly with severity of bone disease and was the only factor independently associated with a greater bone loss over time, may be the most important factor affecting bone mass in patients with PSC also suffering from IBD.

Also, patients with IBD may have low bone mass at presentation before commencement of corticosteroids. ${ }^{4}$ The role of inflammatory cytokines such as interleukin 1 and 6 and tumor necrosis factor in promoting increased bone resorption and decreased bone formation may be one of the critical factors leading to bone disease in patients with $\mathrm{IBD}^{34}$ as is vitamin $\mathrm{D}$ defiency ${ }^{35}$ regardless of corticosteroid use. In addition, vitamin $\mathrm{K}$ has been implicated in promoting bone health in the post-menopausal state, and in a number of clinical conditions associated with bone loss including primary biliary cirrhosis. ${ }^{36,37}$ However, further studies are necessary to determine both the association of bone disease and vitamin $\mathrm{K}$ deficiency in patients with PSC, and what therapeutic effects vitamin K may have in the bone disease in patients with PSC.

It remains uncertain when and how often the bone density measurement needs to be repeated in patients with PSC. Our data can serve as the basis for guidelines regarding the frequency of BMD testing in these patients. Since more than half of patients with PSC present with a tscore in the range of osteopenia or osteoporosis it seems reasonable to measure the bone density in all patients at the time of diagnosis of PSC. Patients with PSC lose bone mass at an average of $1 \%$ per year; this rate of bone loss along with duration of IBD (i.e., the only independent predictor of higher rate of bone loss) should be considered when deciding the frequency of subsequent bone density measurement. For those patients with normal bone mass and short duration of IBD, repeating the BMD every 2 to 3 years seems appropriate. However, repeating the BMD at annual or semiannual intervals seems to be appropriate for those patients with bone mass in the range of osteopenia, particularly those with long-lasting IBD; this would allow the early identification and more aggressive management of the bone disease in those patients progressing to osteoporosis.

There are no data on prevention and treatment of bone disease in patients with PSC. However, general recommendations such as supportive measurements for bone health (i.e., increased physical exercise, and quit alcohol and smoking), and assuring a diet with appropriate content of calcium and vitamin D, or their supplementation if deficient, can be implemented in patients with PSC. ${ }^{38}$ Similarly, there are no data regarding the appropriate time to start treatment for the bone disease in patients with PSC; but patients with established osteoporosis and those who had experienced a fragility fracture should be treated to reduce the risk of further fractures as recommended in the general population.39 In addition, since all fragility fractures in our cohort occurred with a t-score $<-1.5$, it seems reasonable to consider treatment in patients with PSC and BMD below this threshold, 
particularly if they have the risk factors for osteoporosis identified in this study, or if they are on the waiting list for liver transplantation as a significant increment in the risk of fragility fractures is expected in the post-transplant period.10 Although no controlled trials have been reported in PSC, some medications including bisphosphonates, hormone replacement therapy, and selective estrogen receptor modulators, have been evaluated for the treatment of bone disease in patients with $\mathrm{PBC}, 40^{-43}$ and in the liver transplant setting. 44-46 These medications seemed relatively efficacious and safe that they are often used to treat the bone disease in PSC, but data from controlled trials in PSC are needed.

In summary, osteoporosis occurs commonly in patients with PSC. Longer duration of lBD contributes significantly to the severity and rate of progression of bone disease and this effect seems to be independent of other risk factors identified previously in the general population. Our results show that older age, lower BMI and longer duration of IBD help identify the subgroup of patients with PSC with more severe bone disease. This subset of patients would be expected to derive the most benefit from serial measurements of bone density, from adherence to medical therapy and from being enrolled in clinical trials aimed at treating and preventing their bone loss.

\section{Acknowledgments}

Grant support: This study was supported by an R01 DK82426 grant to Dr. Paul Angulo.

\section{Abbreviations}

$\begin{array}{ll}\text { PSC } & \text { primary sclerosing cholangitis } \\ \text { PBC } & \text { primary biliary cirrhosis } \\ \text { IBD } & \text { inflammatory bowel disease } \\ \text { BMI } & \text { body mass index } \\ \text { AST } & \text { aspartate aminotransferase } \\ \text { BMD } & \text { bone mineral density } \\ \text { OR } & \text { odds ratio } \\ \text { CI } & \text { confidence intervals } \\ \text { ROC } & \text { receiver operating characteristics curve }\end{array}$

\section{References}

1. Bonkovsky HL, Hawkins M, Steinberg K, et al. Prevalence and prediction of osteopenia in chronic liver disease. Hepatology 1990;12:273-280. [PubMed: 2391068]

2. Rouillard S, Lane NE. Hepatic osteodystrophy. Hepatology 2001;33:301-307. [PubMed: 11124849]

3. Angulo P, Lindor KD. Primary sclerosing cholangitis. Hepatology 1999;30:325-32. [PubMed: 10385674]

4. Ghosh S, Cowen S, Hannah WJ, et al. Low bone mineral density in Crohn's disease, but not in ulcerative colitis at diagnosis. Gastroenterology 1994;107:1031-1039. [PubMed: 7926456]

5. Vestergaard P, Krogh K, Rejnmark L, et al. Fracture risk is increased in Crohn's disease, but not in ulcerative colitis. Gut 2000;46:176-81. [PubMed: 10644310]

6. Bernstein CN, Seeger LL, Sayre JW, et al. Decreased bone density in inflammatory bowel disease is related to corticosteroid use and not disease diagnosis. J Bone Miner Res 1995;10:250-256. [PubMed: 7754804]

7. Hay EJ, Lindor KD, Wiesner RH, et al. The metabolic bone disease of primary sclerosing cholangitis. Hepatology 1991;14:257-261. [PubMed: 1860683] 
8. Angulo P, Therneau TM, Jorgensen A, et al. Bone disease in patients with primary sclerosing cholangitis: prevalence, severity and prediction of progression. J Hepatol 1998;29:729-35. [PubMed: 9833910]

9. Guichelaar MMJ, Kendall R, Malinchoc M, et al. Bone mineral density before and after OLT: longterm follow-up and predictive factors. Liver Transpl 2006;12:1390-1402. [PubMed: 16933236]

10. Guichelaar MMJ, Schmall J, Malinchoc M, et al. Fractures and avascular necrosis before and after orthotopic liver transplantation: long-term follow-up and predictive factors. Hepatology 2007;46:1198-1207. [PubMed: 17654700]

11. Kim WR, Poterucha JJ, Wiesner RH, et al. The relative role of the Child-Pugh classification and the Mayo natural history model in the assessment of survival in patients with primary sclerosing cholangitis. Hepatology 1999;29:1643-1648. [PubMed: 10347102]

12. Lichtiger S, Present DH, Kornbluth A, et al. Cyclosporine in severe ulcerative colitis refractory to steroid therapy. N Engl J Med 1994;330:1841-1845. [PubMed: 8196726]

13. Best WR, Becktel JM, Singleton JW, et al. Development of a Crohn's disease activity index. National Cooperative Crohn's disease study. Gastroetnerology 1976;70:439-444.

14. Angulo P, Batts KP, Jorgensen RA, et al. Oral budesonide in the treatment of primary sclerosing cholangitis. Am J GastroenteroI 2000;95:2333-2337.

15. World Health Organization. Technical report no 843. Genova: World Health Organization; 1994. Assessment of fracture risk and its application to screening for postmenopausal osteoporosis.

16. Kanis JA, Melton J III, Christiansen C, et al. The diagnosis of osteoporosis. J Bone Miner Res 1994;9:1137, 114-116. [PubMed: 7976495]

17. Eastell R. Treatment of postmenopausal osteoporosis. N Engl J Med 1998;338:736-746. [PubMed: 9494151]

18. Wahner, HW.; Folgelman, I. The evaluation of osteoporosis: dual energy X ray absorptiometry in clinical practice. Martin Dunitz Ltd; London: 1994.

19. Kelly TL. Bone mineral density reference database for American men and women. J Bone Min Res 1990;5(suppl 2):S249.

20. Kroger H, Tuppurainen M, Honkanen R, et al. Bone mineral density and risk factors for osteoporosis-a population-based study of 1600 perimenopausal women. Calcif Tissue Int 1994;55:1-7. [PubMed: 7922782]

21. Felson DT, Zhang Y, Hannan MT, et al. Effect of weight and body mass index on bone mineral density in men and women: the Framingham study. J Bone Miner Res 1993;8:567-573. [PubMed: 8511983]

22. Tremollieres FA, Pouilles IM, Ribot C. Vertebral postmenopausal bone loss is reduced in overweight women: a longitudinal study in 155 early postmenopausal women. J Clin Endocrinol Metab 1993;77:683-686. [PubMed: 8370689]

23. Ducy P, Amling M, Takeda S, et al. Leptin inhibits bone formation through a hypothalamic relay: a central control of bone mass. Cell 2000;100:197-207. [PubMed: 10660043]

24. Wiesner RH, LaRussi NF. Clinicopathologic features of the syndrome of primary sclerosing cholangitis. Gastroenterology 1980;79:200-206. [PubMed: 7399227]

25. Menon KV, Angulo P, Weston S, et al. Bone disease in primary biliary cirrhosis: independent indicators and rate of progression. J Hepatol 2001;35:316-323. [PubMed: 11592591]

26. Newton J, Francis R, Prince M, et al. Bone disease in parimary biliary cirrhosis revisited. Gut 2001;49:282-287. [PubMed: 11454807]

27. Springer JE, Cole DE, Rubin LA, et al. Vitamin D-receptor genotypes as independent genetic predictors of decreased bone mineral density in primary biliary cirrhosis. Gastroenterology 2000;118:145-151. [PubMed: 10611163]

28. Guanabens N, Cerda D, Monegal A. Low bone mass and severity of cholestasis affect fracture risk in patients with primary biliary cirrhosis. Gastroenterology 2010;138:2348-2356. [PubMed: 20178794]

29. Janes CH, Dickson ER, Okazaki R, et al. Role of hyperbilirubinemia in the impairment of osteoblast proliferation associated with cholestatic jaundice. J Clin lnvest 1995;95:2581-6. 
30. Guichelaar MM, Malinchoc M, Sibonga J, et al. Bone metabolism in advanced cholestatic liver disease: analysis by bone histomorphometry. Hepatology 2002;36:895-903. [PubMed: 12297836]

31. Lundqvist K, Broome U. Differences in colonic disease activity in patients with ulcerative colitis with and without primary sclerosing cholangitis: a case control study. Dis Colon Rectum 1997;40:451-456. [PubMed: 9106695]

32. Compston JE, Judd D, Crawley EO, et al. Osteoporosis in patients with inflammatory bowel disease. Gut 1987;28:410-415. [PubMed: 3583068]

33. Abitol V, Roux C, Chaussade S, et al. Metabolic bone assessment of patients with inflammatory bowel disease. Gastroenterology 1995;108:417-422. [PubMed: 7835582]

34. Monologies SC, Jika RL. Bone marrow cytokine and bone remodeling: emerging insights in the pathophysiology ofosteoporosis. N Engl J Med 1995;332:305. [PubMed: 7816067]

35. Valentine JF, Sninsky. Prevention and treatment of osteoporosis in patients with inflammatory bowel disease. Arn J Gastroenterol 1999;94:878-883.

36. Plaza SM, Lamson DW. Vitamin K2 in bone metabolism and osteoporosis. Altern Med Rev 2005;10:24-35. [PubMed: 15771560]

37. Nishiguchi S, Shimoi S, Kurooka H, et al. Randomized pilot trial of vitamin K2 for bone loss in patients with primary biliary cirrhosis. J Hepatol 2001;35:543-545. [PubMed: 11682046]

38. NIH Consensus Development Conference Statement. Osteoporosis Prevention, Diagnosis, and Therapy. Bethesda, MD: National Institute of Health; 2000 [28 September 2010]. Access at http://consensus.nih.gov/2000/2000Osteoporosis111html.htm on

39. Qaseem A, Snow V, Shekelle P, et al. Pharmacologic treatment of low bone density or osteoporosis to prevent fractures: a clinical practice guideline from the American college of physicians. Ann Intern Med 2008;149:404-415. [PubMed: 18794560]

40. Zein CO, Jorgensen RA, Clarke B, et al. Alendronate improves bone mineral density in primary biliary cirrhosis: A randomized placebo-controlled trial. Hepatology 2005;42:762-771. [PubMed: 16175618]

41. Kaemmerer D, Lehmann G, Wolf G, et al. Treatment of osteoporosis after liver transplantation with ibandronate. Transpl Int 2010;23:753-759. [PubMed: 20158691]

42. Menon K, Angulo P, Boe GM, et al. Safety and efficacy of estrogen therapy in preventing bone loss in primary biliary cirrhosis. Am J Gastroenterol 2003;98:889-892. [PubMed: 12738473]

43. Levy C, Harnois DM, Angulo P, et al. Raloxifene improves bone mass in osteopenic women with primary biliary cirrhosis: results of a pilot study. Liver Int 2005;25:117-121. [PubMed: 15698408]

44. Vedi S, Ninkovic M, Garrahan NJ, et al. Effects of a single infusion of pamidronate prior to liver transplantation: a bone histomorphometric study. Transpl Int 2002;15:290-295. [PubMed: 12072899]

45. Monegal A, Guanabens N, Suarez MJ, et al. Pamidronate in the prevention of bone loss after liver transplantation: a randomized controlled trial. Transpl Int 2009;22:198-206. [PubMed: 18796000]

46. Misof BM, Bodingbauer M, Roschger P, et al. Short-term effects of high-dose zoledronic acid treatment on bone mineralization density distribution after orthotopic liver transplantation. Calcif Tissue Int 2008;83:167-175. [PubMed: 18712431] 


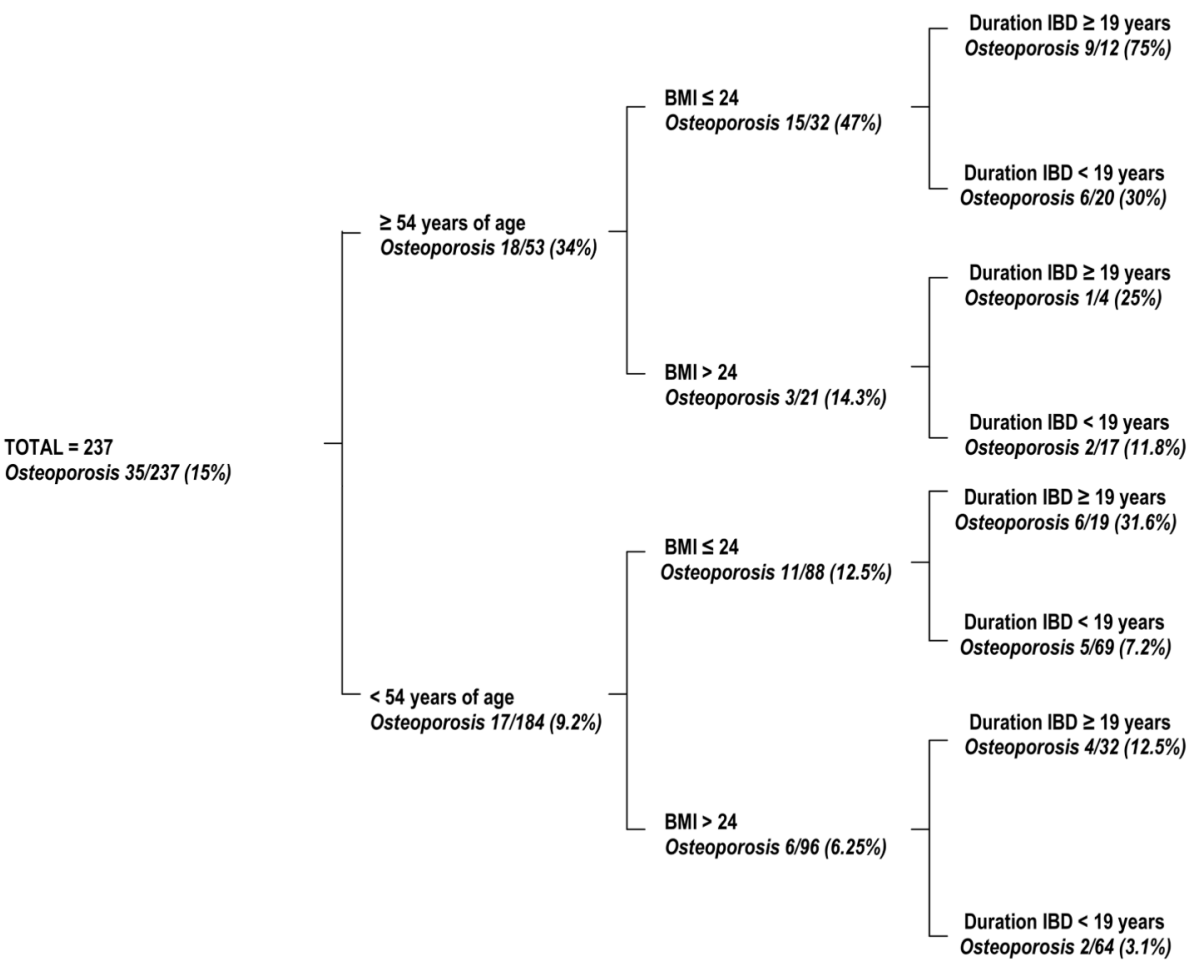

FIGURE 1.

Decision tree using the three variables (age, body mass index, and duration of IBD)

identified by multivariate analysis. The term osteoporosis refers to a t-score below -2.5 

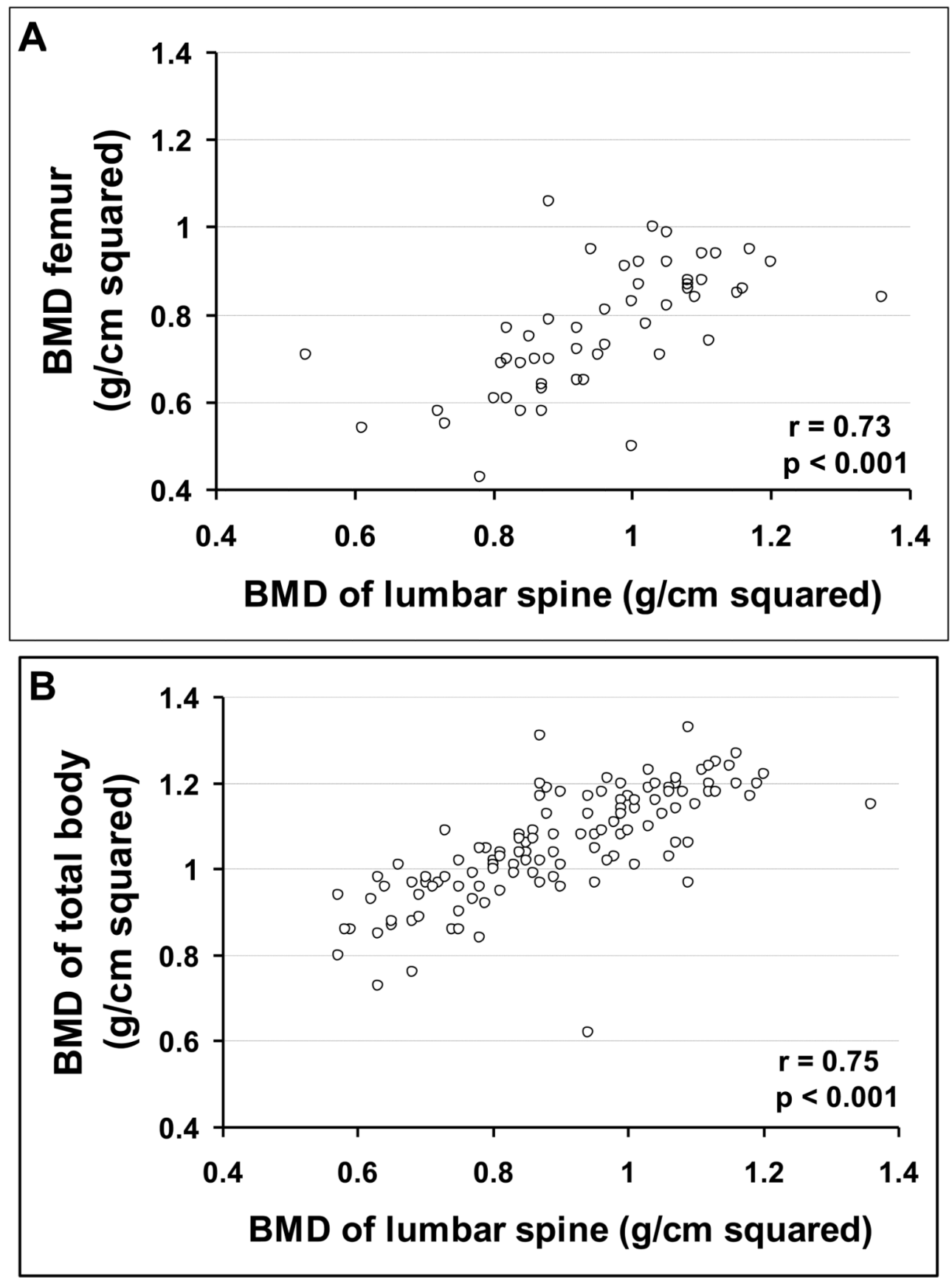

FIGURE 2.

Correlation of bone mineral density of the lumbar spine (L2-L4) with (A) bone mineral density of femoral neck, and (B) bone mineral density of total body. 


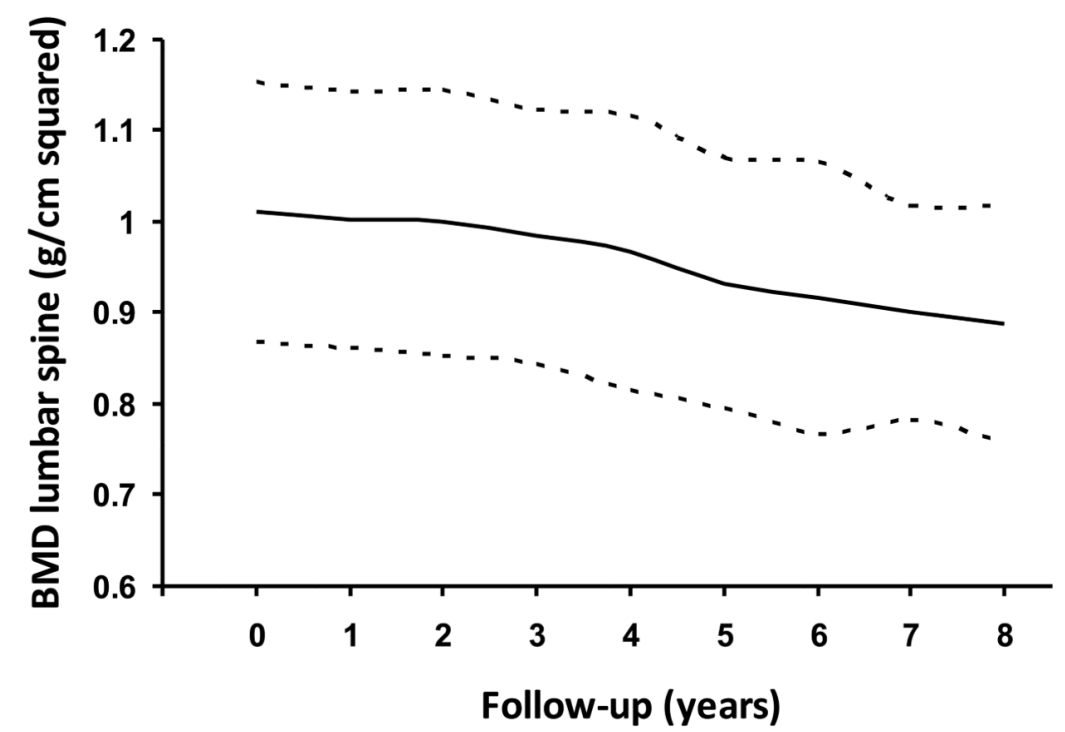

FIGURE 3.

Bone mineral density of the lumbar spine (L2-L4) at baseline and at annual intervals. The continuous line refers to mean and the discontinuous line refers to standard error of the mean. 


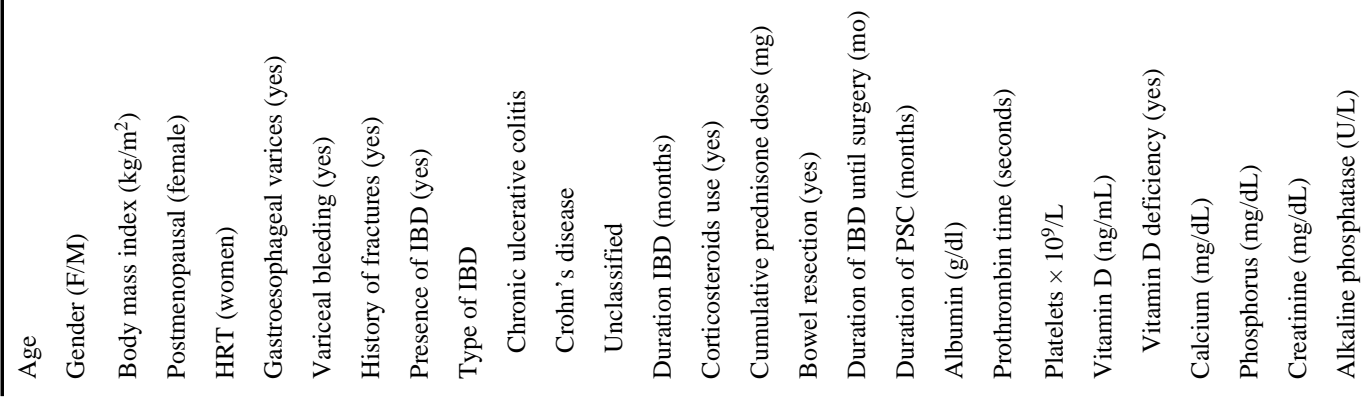




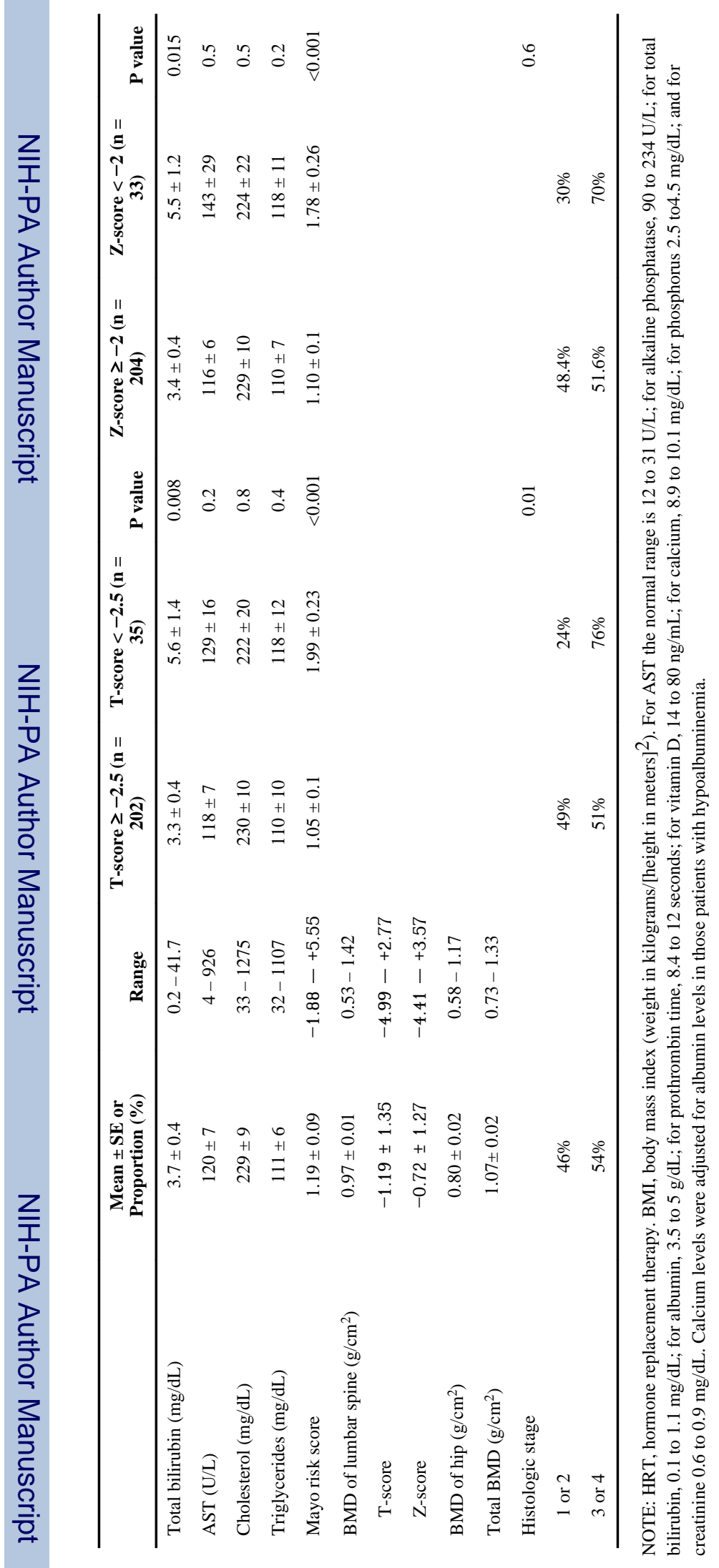


Table 2

Multivariate analysis of the association between osteoporosis $(\mathrm{T}$-score $<-2.5)$ and independent predictors

\begin{tabular}{|c|c|c|c|c|}
\hline & \multicolumn{4}{|c|}{ 95\% Confidence Intervals } \\
\hline & Odds Ratio & Lower & Upper & P value \\
\hline \multicolumn{5}{|c|}{ Age (years) } \\
\hline$<54$ & 1 & & & \\
\hline$\geq 54$ & 7.8 & 3.3 & 18.3 & $<0.001$ \\
\hline \multicolumn{5}{|c|}{ Body mass index } \\
\hline$>24$ & 1 & & & \\
\hline$\leq 24$ & 4.9 & 1.9 & 12.6 & 0.001 \\
\hline \multicolumn{5}{|c|}{ Duration of IBD (years) } \\
\hline$<19$ & 1 & & & \\
\hline$\geq 19$ & 3.6 & 1.5 & 8.4 & 0.004 \\
\hline
\end{tabular}


Table 3

Multivariate analysis of the association between severe osteoporosis (Z-score $<-2.0)$ and independent predictors

\begin{tabular}{|c|c|c|c|c|}
\hline & \multirow[b]{2}{*}{ Odds Ratio } & \multicolumn{2}{|c|}{$95 \%$ Confidence Interval } & \multirow[b]{2}{*}{$P$ value } \\
\hline & & Lower & Upper & \\
\hline \multicolumn{5}{|c|}{ Body mass index } \\
\hline$>24$ & 1 & & & \\
\hline$\leq 24$ & 7.5 & 2.0 & 27.7 & 0.003 \\
\hline \multicolumn{5}{|c|}{ Duration of IBD (years) } \\
\hline$<19$ & 1 & & & \\
\hline$\geq 19$ & 3.7 & 1.3 & 10.1 & 0.01 \\
\hline
\end{tabular}

